\section{A boy with recurrent swelling of the jaw}

\author{
Lien Haverals, ${ }^{1}$ Marjolein Mattheij, $, 1,2$ \\ Esther Hoppenreijs, ${ }^{3}$ Stefaan Bergé, ${ }^{4}$ \\ Annemieke van der Weij ${ }^{2}$ \\ 1 Emergency Department, Antwerp \\ University Hospital, Belgium; \\ 2Department of Pediatrics, Jeroen Bosch \\ Hospital, 's-Hertogenbosch, The \\ Netherlands; ${ }^{3}$ Department of Pediatric \\ Rheumatology, University Medical \\ Centre Nijmegen, The Netherlands; \\ ${ }_{4}^{4}$ Department of Oral Surgery, University \\ Medical Centre Nijmegen, The \\ Netherlands
}

\begin{abstract}
We present a 10-year old boy with recurrent swelling of the right mandibular region. Based on the diagnosis of chronic recurrent parotitis, he received only supportive treatment. Because of frequent relapses, the diagnosis was reconsidered. Magnetic resonance imaging, scintigraphy and biopsy were compatible with chronic osteomyelitis. This lead to a diagnosis of mandibular primary chronic osteomyelitis, an uncommon non-suppurative inflammatory disease of unknown origin. After decortication of the mandible, the patient recovered well. Because there were no further complaints, the follow-up was ended 18 months after the operation.
\end{abstract}

\section{Introduction}

Primary chronic osteomyelitis of the jaw is a rare, non-suppurative, chronic inflammation of unknown etiology. It causes periodic episodes of mandibular pain, swelling in the lower jaw region and paresthesia over the affected areas. The disease is characterized by lack of pus formation, fistula or formation of bone sequestra. It is reported in patients of both sexes with a peak onset between 10 and 20 years. We report a case of a ten-year-old boy who presented with recurrent unilateral swelling of the right jaw.

\section{Case Report}

A ten-year-old boy presented for a second opinion to the pediatric outpatient clinic with complaints of periodic swelling and pain in the right jaw region. Since two years, periodic swelling occurred every other month and remained present for several days to weeks. Consumption of acid fluids was painful. No fever was noted. During earlier acute phases, antibiotics had been administered twice, with a positive effect on the duration of the symptoms. Earlier ultrasound examination, dynamic magnetic resonance imaging (MRI) and sialography at the time of the swelling did not reveal any abnormalities, although his parents reported that he remained without symptoms for three months after the sialography, which was longer than normal. Medical and family history revealed no relevant facts, especially no history of autoimmune disease. The boy was vaccinated according to the Dutch vaccination program.

Because of the frequent and recurrent character of the symptoms, the parents were seeking a second opinion after an earlier diagnosis of chronic recurrent parotitis. When he presented at the outpatient clinic, swelling and pain of the right jaw region had been present for one month (Figure 1).

Physical examination did not reveal abnormalities in the mouth and ears, except for the fact that he was not able to fully open his mouth (trismus). There were no signs of infection, periodontitis, caries or purulent saliva discharge in the mouth. Blood analysis showed an elevated erythrocyte sedimentation rate (ESR), and a normal amylase. The first echography performed during complaints showed lymphadenitis, without parotitis, while a second echography, also during complaints, showed a parotitis. The results of the examinations are listed in Table 1. As not all examinations were compatible with parotitis, and as there was no explanation for the fact that the boy was unable to fully open his mouth, a new MRI of the jaw was performed. This showed a diffusely broadened mandible, several areas of interruption of the cortex as well as soft tissue swelling (Figure 2A), which was considered to be compatible with osteomyelitis. A technetium scan confirmed the diagnosis of osteomyelitis (Figure 2B), while a skeletal scintigraphy showed no other foci (Figure 2C). Biopsy showed signs of remodeling with fibrosis, consistent with chronic osteomyelitis. These examinations led to diagnosis of mandibular primary chronic osteomyelitis (MPCO).

A decortication was performed (Figure 3). After surgery, the boy did not report any further complaints or relapses.
Correspondence: Marjolein Mattheij, Antwerp University Hospital, Wilrijkstraat 10, 2650 Edegem, Belgium.

Tel.: +323.8214144

E-mail: marjolein.mattheij@uza.be

Key words: Chronic osteomyelitis; mandibular primary chronic osteomyelitis (MPCO); chronic recurrent parotitis.

Acknowledgments: We would like to thank our patient and his parents for giving us permission to show clinical pictures.

Contributions: LH, MM: writing of manuscript. LH, MM, EH, SB, AW: collecting medical information, analyzing and selecting investigations; SB: performing the surgery, providing pictures of surgical procedure; $\mathrm{LH}, \mathrm{MM}, \mathrm{AW}$ : manuscript writing; $\mathrm{EH}, \mathrm{SB}$ : manuscript reviewing.

Conflict of interests: the authors declare no conflict of interests.

Received for publication: 11 November 2017. Accepted for publication: 17 November 2017.

This work is licensed under a Creative Commons Attribution NonCommercial 4.0 License (CC BY-NC 4.0)

CCopyright L. Haverals et al., 2017

Licensee PAGEPress, Italy

Pediatric Reports 2017; 9:7489

doi:10.4081/pr.2017.7489

\section{Discussion}

This case report illustrates the difficulties encountered during diagnosing a child with recurrent swelling in the jaw region. In the presented case an auto-inflammatory disease (chronic recurrent multifocal osteomyelitis), autoimmune, obstructive or chronic recurrent parotitis, as well as (non-) ossifying fibroma, fibrous dysplasia, lymphadenitis or secondary lymphadenopathy due to malignancy such as osteosarcoma were considered. The location of the symptoms and the positive impact of sialography were concomitant with the diagnosis of chronic recurrent parotitis, but the presence of atypical findings (normal values of serum amylase, long duration of the symptoms and difficulty in opening the mouth) made us doubt the earlier diagnosis.

Chronic recurrent parotitis is characterized by recurrent episodes of swelling and pain of unknown etiology in the parotid gland. ${ }^{1}$ Generally, complaints start at the age of five, and virtually all patients become asymptomatic by the age of 10-15 years. The duration of symptoms averages between 3-7 days but may last 2-3 weeks in 
some individuals. In this case, however, symptoms lasted significantly longer. Recurrent parotitis can be a sign of a primary Sjögren disease in children, but this patient did not show other symptoms of Sjögren's disease.

Because of the earlier normal MRI investigation of the jaw, bone abnormalities were initially not considered. This MRI, however, was a dynamic investigation to evaluate luxation of the jaw joint in motion, which is not specifically aimed at detecting osteomyelitis. This emphasizes the complexity of interpreting MRI images in children and the need to focus a specific question. Retrospectively, a different focus while evaluating the images of the first MRI may have led to a different interpretation. Besides the presence of contradicting findings during the diagnostic phase, the difficulty of the diagnosis can also be linked to the rarity of the disease of MPCO.

Osteomyelitis can be defined as an inflammatory state of cortical and cancellous bone, whereby the most affected sites are the long bones of extremities.2,3 Mandibular osteomyelitis can be either acute or chronic. In cases with a duration of less than four weeks, the term acute is used, while chronic is considered when no acute status is observed with symptoms appearing periodically with different intensity and duration.

In mandibular chronic osteomyelitis two major forms occur: suppurative and non-suppurative forms. Non-suppurative (called primary) mandibular osteomyelitis (MPCO) is characterized by the absence of pus formation, fistula and sequestration. Suppurative (called secondary) mandibular osteomyelitis is more common than MPCO and is mainly caused by odontogenic infections occurring as a complication of dental extractions, maxillofacial trauma or irradiation of facial skeleton. Typical for suppurative mandibular osteomyelitis (MSCO) is

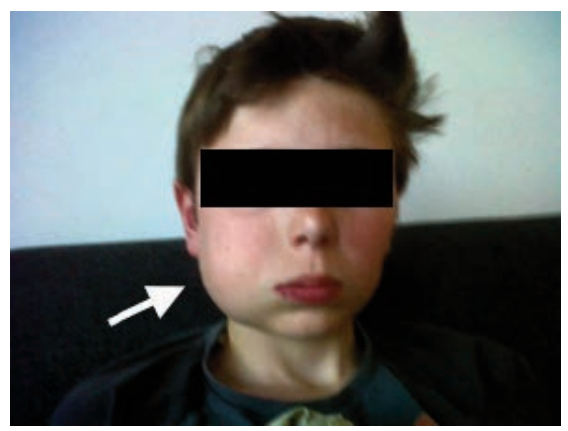

Figure 1. Photograph of the boy at the time of the swelling (white arrow). the presence of pus formation, fistula and sequestration. 3,4 The importance of a maxillofacial trauma can be debated in this case. Retrospectively, the parents reported that the boy suffered from a sport trauma where a hockey ball hit his jaw, which could be an indication towards secondary mandibular osteomyelitis. However, in this case, no pus formation, fistula or sequestration were found. This made us conclude that the presentation of our patient was most compatible with the diagnosis of MCPO.

MPCO is only described in some case reports or small case studies.3,7 It occurs in all age groups of both genders, but clinical symptoms are generally more prominent in younger patients, and become less prominent as the disease progresses. ${ }^{5}$ Although MPCO has a peak onset between 10 and 20 years of age, ${ }^{6}$ most published data are focused on adult patients, making the disease even more unknown to pediatric specialists. ${ }^{6-8}$ MPCO causes mandibular pain, swelling in the lower jaw region, decreased inferior alveolar nerve sensation or paresthesia over affected areas and/or lower lip (Vincent's symptom), restrictions in mouth opening and regional lymphadenopathy. ${ }^{3}$ With regard to the maxillofacial region, PCO almost exclusively affects the mandible, ${ }^{10}$ since the collateral blood supply of the mandible is poor compared to the

Table 1. Examinations.

\begin{tabular}{|c|c|c|}
\hline Investigation & Result & Interpretation \\
\hline ESR & $50 \mathrm{~mm} / \mathrm{h}$ & Elevated, normal: $<15 \mathrm{~mm} / \mathrm{h}$ \\
\hline $\mathrm{Hb}$ & $7.0 \mathrm{mmol} / \mathrm{L}$ & Normal \\
\hline Thrombocytes & $214 \times 10^{\circ} 9 / \mathrm{L}$ & Normal \\
\hline Leucocytes & $6.4 \times 10^{\circ} 9 / \mathrm{L}$ & Normal \\
\hline CRP & $6 \mathrm{mg} / \mathrm{L}$ & Normal \\
\hline Amylase & $77 \mathrm{u} / \mathrm{L}$ & Normal \\
\hline ANA & Positive, titer 1:80 & Low titer \\
\hline ENA & Negative & Normal \\
\hline $\operatorname{IgE}$ & $16 \mathrm{U} / \mathrm{L}$ & Normal \\
\hline Paramyxovirus & IgG positive, IgM negative & Normal post vaccination \\
\hline Bartonella henselae & IgM negative & Normal \\
\hline Toxoplasma & IgG negative, IgM negative & No exposure \\
\hline EBV & IgG positive, IgM negative & No current infection \\
\hline CMV & IgG negative, IgM negative & No exposure \\
\hline Chest X-ray & Negative & Normal \\
\hline Mantoux & Negative & Normal \\
\hline Echography jaw/neck (1) & Lymphadenitis & \\
\hline Echography jaw/neck (2) & Parotitis & \\
\hline Examination ORL & Normal & Normal \\
\hline
\end{tabular}

ESR, erythrocyte sedimentation rate; Hb, hemoglobin; CRP, C-reactive protein; ANA, anti-nuclear antibody; ENA, extractable nuclear antigens antibody; IgE, Immunoglobulin E; IgG, Immunoglobulin G; IgM, Immunoglobulin M; EBV, Epstein-Barr virus; CMV, cytomegalovirus; ORL, otorhinolaryngology.

\section{upper jaw. ${ }^{8}$}

The pathogenesis of MPCO remains unclear. ${ }^{6}$ Since no evidence for a direct infectious etiology was ever found, infection is unlikely to be an etiologic factor. 3,8 Hypotheses for etiology include bacterial infection of dental origin or more distant foci, autoimmunity, auto-inflammatory, or chronic tendoperiostitis. ${ }^{5}$ An association between MPCO and dermato-skeletal disorders such as SAPHO (synovitis, acne, pustulosis, hyperostosis and osteitis), CRMO (chronic recurrent multifocal osteomyelitis) or Majeed syndrome has been suggested.3,5,11 In our case, skeletal scintigraphy did not show other foci, justifying the decision to abandon the diagnosis of CRMO. Theoretically however it cannot be excluded that MPCO could be an unifocal manifestation of chronic non-bacterial osteomyelitis (CNO) or first manifestation of CRMO. ${ }^{12,13}$ The patient did not have synovitis, pustulosis or acne and did not classify for SAPHO.

In MPCO, laboratory findings are nonspecific. Sedimentation rate and C-reactive protein level can be slightly increased, but they can also be normal. Specific biomarkers are not yet available for this diagnosis. Several imaging techniques have been used for diagnosis, including panoramic radiograph, MRI, skeletal scintigraphy and CT- 

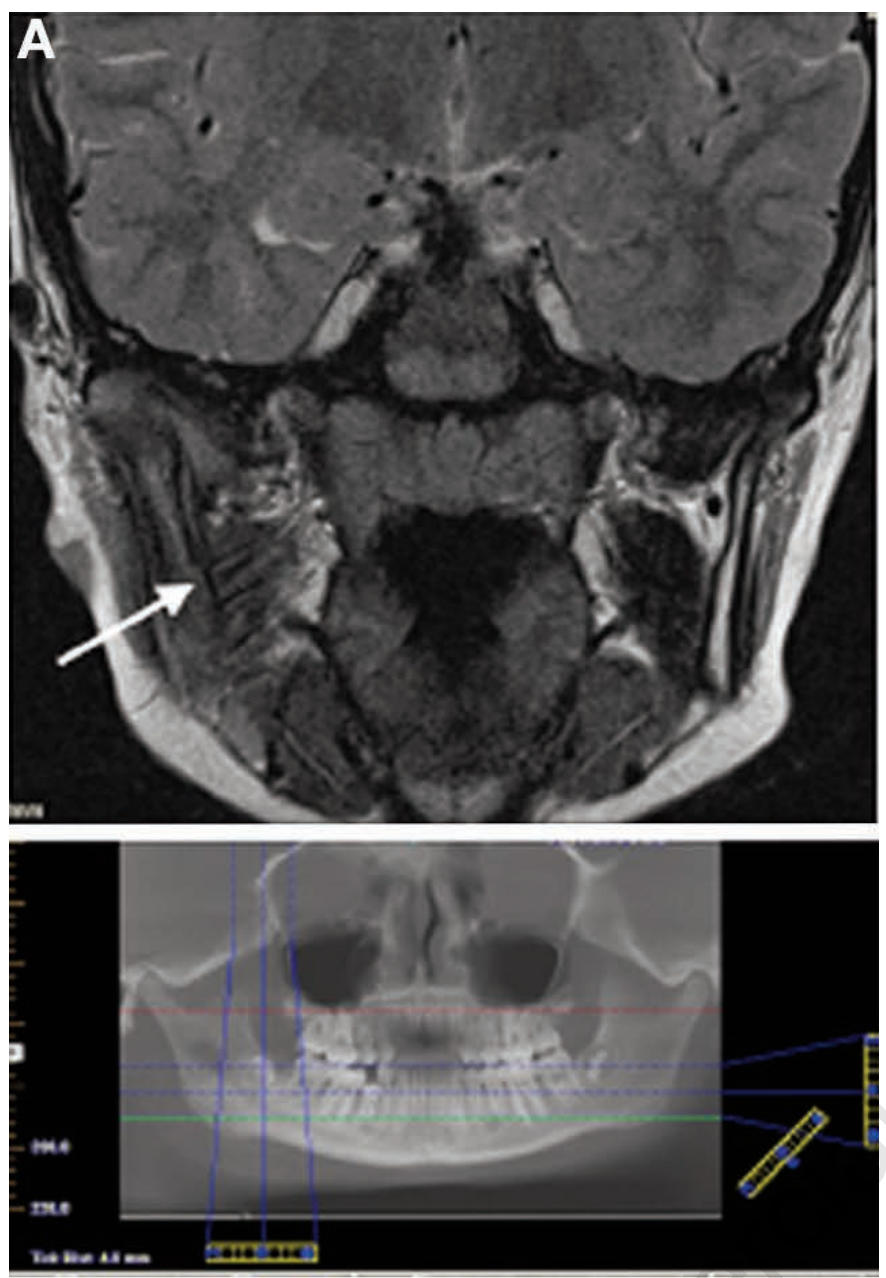

B
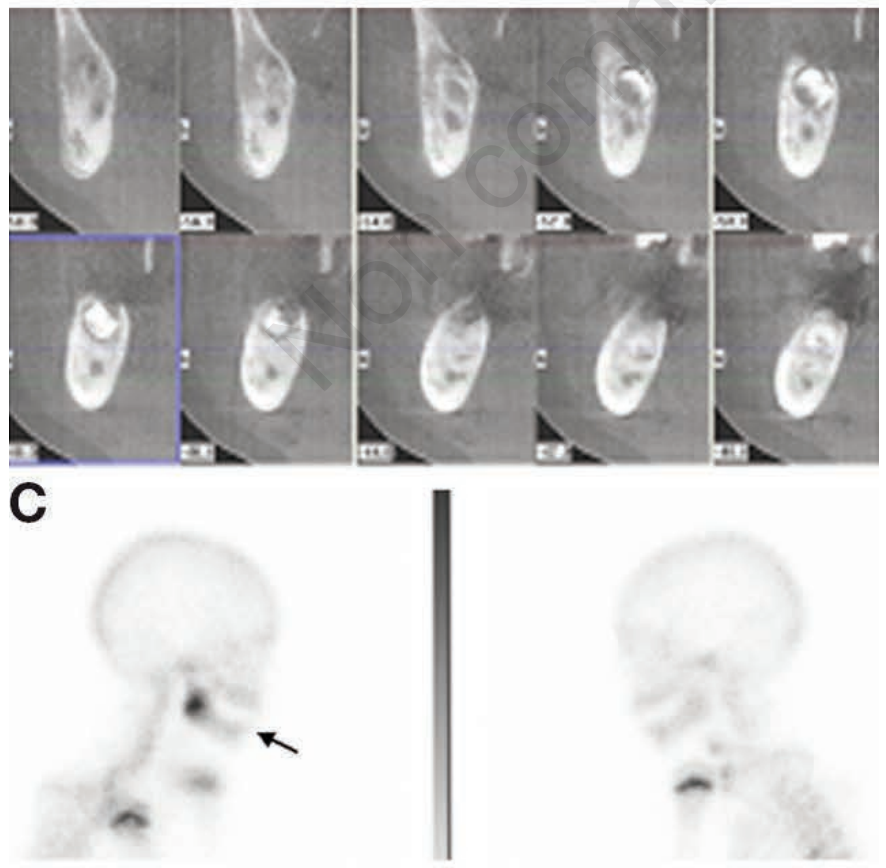

Figure 2. Investigations of the jaw: A) Magnetic Resonance Imaging (MRI) performed two years after start of symptoms. The white arrow indicates where the right mandible is diffusely broadened, with several areas of interruption of the cortex and soft tissue swelling. B) Technetium (TC) scan. C) Bone scintigraphy of the patient shows a significant enhancement of the right side of the mandible (black arrow). scan, as they can be characteristic. 11

Several therapeutic options for MPCO with varying effectiveness and evidence have been suggested. Although infection remains unproven as a cause of MPCO, antibiotics are mentioned as a possible therapy.3,5 Non-steroidal anti-inflammatory drugs and corticosteroids are reported to reduce symptoms such as swelling and trismus. Secondary options are methotrexate, anti-tumor necrosis factor, bisphosphonate or sulfasalazine. ${ }^{3,5}$ In children with CRMO with mandibula involvement there are good results with bisphosphonates and anti-TNF treatment. 13

Several authors mention the importance of removal of necrotic bone tissue (decortication) in MPCO, although in children disturbance of mandible growth has been reported.14 Hyperbaric oxygen therapy (HBOT), where higher levels of available oxygen cause induction of capillary formation and increased activity of leukocytes, has also been used in children with MPCO. 15,16

Due to the small number of patients with this disease and the fluctuant natural course of the disease, conclusive recommendations as to the best therapeutic option are difficult to make. The long-term prognosis is moderate: although recurrences are frequently described, complete cure has also occurred spontaneously after a prolonged clinical course. 6
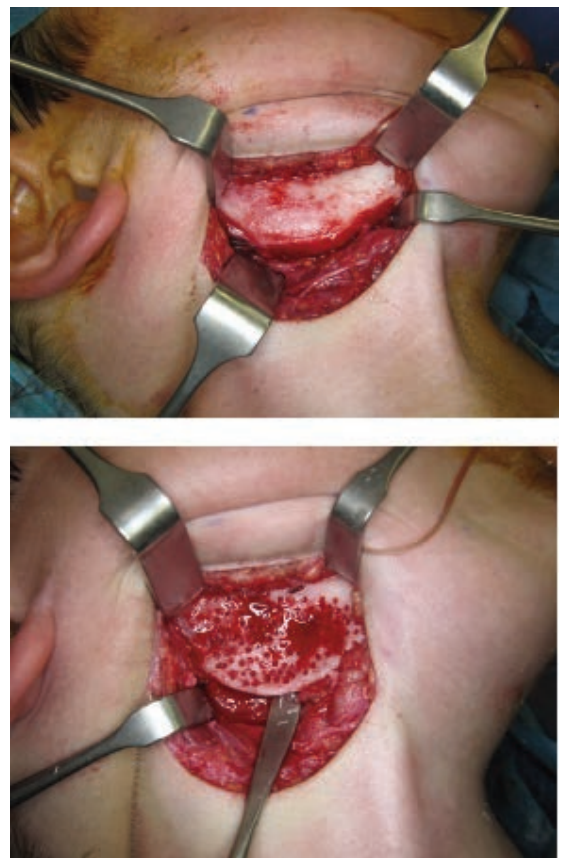

Figure 3. Perioperative imaging of decortication. 


\section{Conclusions}

Although the supposed diagnosis of chronic recurrent parotitis was based on a series of clinical findings and examinations, some findings were not concomitant with this disease. In this case, after complaints for 2 years, a fresh view has led to the diagnosis of MPCO.

The history of this case shows that the question asked while performing an investigation can influence the interpretation of the test. This is an important lesson to learn. When signs and symptoms do not fit the assumed diagnosis, re-evaluation of previous images and investigations, performing additional investigations and considering rare diseases can be helpful.

\section{References}

1. Mahalakshmi S, Kandula S, Shilpa P, Kokila G. Chronic recurrent non-specific parotitis: a case report and review. Ethiopian J Health Sci 2017;27:95-100.

2. Stern SM, Ferguson PJ. Autoinflammatory bone diseases. Rheum Dis Clin North Am 2013;39: 735-49.

3. Berglund C, Ekstromer K, Abtahi J. Primary chronic osteomyelitis of the jaws in children: an update on patho- physiology, radiological findings, treatment strategies, and prospective analysis of two cases. Case Rep Dent 2015;2015:152717.

4. Agarwal A, Kumar N, Tyagi A, De N. Primary chronic osteomyelitis in the mandible: a conservative approach. BMJ Case Rep 2014;2014.

5. Baltensperger M, Gratz K, Bruder E, et al. Is primary chronic osteomyelitis a uniform disease? Proposal of a classification based on a retrospective analysis of patients treated in the past 30 years. $\mathrm{J}$ Cranio-Maxillo-Fac Surg 2004;32:4350.

6. Theologie-Lygidakis N, Schoinohoriti O, Iatrou I. Surgical management of primary chronic osteomyelitis of the jaws in children: a prospective analysis of five cases and review of the literature. Oral Maxillofac Surg 2011;15:41-50.

7. Eyrich GK, Baltensperger MM, Bruder E, Graetz KW. Primary chronic osteomyelitis in childhood and adolescence: a retrospective analysis of 11 cases and review of the literature. J Oral Maxillofac Surg 2003;61:561-73.

8. Bevin CR, Inwards CY, Keller EE. Surgical management of primary chronic osteomyelitis: a long-term retrospective analysis. J Oral Maxillofac Surg 2008;66:2073-85.

9. Gentry LO. Osteomyelitis: options for diagnosis and management. J Antimicrob Chemother 1988;21:115-
31.

10. Obel G, Krogdahl A, Thygesen T, Godballe C. Juvenile mandibular chronic osteomyelitis: 3 cases and a literature review. J Oral Maxillofac Surg 2013;71:305-9.

11. Petrikowski CG, Pharoah MJ, Lee L, Grace MG. Radiographic differentiation of osteogenic sarcoma, osteomyelitis, and fibrous dysplasia of the jaws. Oral Surg Oral Med Oral Pathol Oral Radiol Endod 1995;80:744-50.

12. Bocchialini G, Ferrari L, Rossini M, et al. Chronic nonbacterial osteomyelitis involving the mandible: a case report. Int J Surg Case Rep 2017;37:149-53.

13. Kaiser D, Bolt I, Hofer M, et al. Chronic nonbacterial osteomyelitis in children: a retrospective multicenter study. Pediatr Rheumatol Online J 2015;13:25.

14. Chrcanovic BR. Open versus closed reduction: mandibular condylar fractures in children. Oral Maxillofac Surg 2012;16:245-55.

15. Lentrodt S, Lentrodt J, Kubler N, Modder U. Hyperbaric oxygen for adjuvant therapy for chronically recurrent mandibular osteomyelitis in childhood and adolescence. J Oral Maxillofac Surg 2007;65:186-91.

16. Fang RC, Galiano RD. Adjunctive therapies in the treatment of osteomyelitis. Semin Plastic Surg 2009;23:141-7. 